\title{
A qualitative study of the stigmatization and coping mechanisms among pregnant teenagers living with HIV in Thailand
}

\author{
Supalak Phonphithak and Narin Hiransuthikul \\ Department of Preventive and Social Medicine, Faculty of Medicine, \\ Chulalongkorn University, Bangkok, Thailand \\ Penchan Pradubmook Sherer \\ Department of Society and Health, Faculty of Social Science and Humanities, \\ Mahidol University, Bangkok, Thailand, and \\ Sasithorn Bureechai \\ Nurse Department, The HIV Netherlands Australia \\ Thailand Research Collaboration, Bangkok, Thailand
}

\begin{abstract}
Purpose - This aim of this study was to explore the experiences of stigmatization and coping mechanisms during pregnancy among pregnant women who are living with HIV in Thailand. The secondary objective was to determine factors contributing to stigma during motherhood among HIV-infected women as well as explore how they cope with the discrimination from society.

Design/methodology/approach - Qualitative data were collected using in-depth interviews to obtain different versions of stigmatized experience from 16 pregnant women living with HIV on stigmatization and coping mechanisms. There were 5 pregnant adolescents living with HIV and 11 adult pregnant women living with HIV. The content analysis was used to examine patterns of stigmatizations and attributed factors.

Findings - Personal stigma was found among pregnant women living with HIV regardless of age. HIV status disclosure was the crucial barrier of accessing to care for people experiencing stigmatizations. Personal stigma associated with higher HIV status was not disclosed. Interestingly, all teenage mothers who participated in this study disclosed their HIV-status to their family. People who have social support especially from family and significant others are found to be able to cope and get through the difficulties better than those who lack those social support.

Originality/value - This study yields outcomes similar to several other studies that have been conducted either in Thailand or other countries. This study found that family support was crucial in reducing HIV stigma. Furthermore, HIV-infected pregnant female adults were more afraid to disclose their HIV status to their husbands, other family members and their work colleagues.
\end{abstract}

Keywords Stigmatization, Teenage pregnancy, Pregnant women living with HIV, Thailand

Paper type Research paper

(c) Supalak Phonphithak, Narin Hiransuthikul, Penchan Pradubmook Sherer and Sasithorn Bureechai. Published in Journal of Health Research. Published by Emerald Publishing Limited. This article is published under the Creative Commons Attribution (CC BY 4.0) licence. Anyone may reproduce, distribute, translate and create derivative works of this article (for both commercial and non-commercial purposes), subject to full attribution to the original publication and authors. The full terms of this licence may be seen at http://creativecommons.org/licences/by/4.0/legalcode

This paper was completed with the support of Chulalongkorn University (grant number.44, 3/2562). The authors wish to thank all the women living with HIV for their participation in this study. The authors are also grateful to all staff members at Chulalongkorn Hospital, HIV-NAT and the Thai Red Cross AIDS Research Center for their contribution to this research.

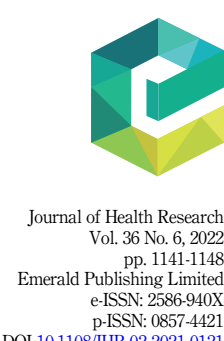

DOI 10.1108/JHR-02-2021-0121 
JHR

36,6

\section{Introduction}

Even though advanced medical technology has the potential to prolong the lifespan of individuals living with HIV, it cannot protect them from discrimination and stigmatization. Interestingly, research has found that gender is related to HIV-related stigma experiences among people living with HIV (PLWHIV). Specifically, females are more likely to be discriminated against than their male counterparts by approximately $50 \%$ [1]. This ongoing phenomenon has raised concerns over the negative impacts felt by pregnant women living with HIV and their newborn babies $[2,3]$. To exacerbate the problem, HIV-positive pregnant women who decide to conceal their HIV status from their partners place themselves at greater risk of discrimination and stigmatization [4], which can have adverse effects on their physical and emotional growth during the transition from adolescence to adulthood. This study found that teenage pregnant participants showed signs of physical and mental problems, including alcohol abuse, lack of knowledge, feelings of hopelessness and low self-value. Another study by Mchunu et al. [5] suggested that pregnant teenagers could be affected by both internal factors (lack of knowledge to prevent pregnancy) and external factors (social and familial discrimination).

In terms of discrimination during teenage pregnancy, a study by Paudel and Baral [6] on knowledge and perception among HIV-infected women revealed that the most sensitive issue to be discussed with young women is HIV-status disclosure, which introduces difficulties dealing with stigmatization from the public eye. However, support from family and the community helps these individuals get through difficult times. Conversely, a study by Simbayi et al. [7] conducted in Cape Town showed that HIV-infected pregnant women with an extremely high degree of self-stigmatization were more likely to be depressed and coped by using drugs.

A study on HIV-pregnant and postpartum women found an association between overwhelming stigmatization and depression, including social isolation among HIV-infected pregnant women, which persists even after childbirth [8]. This implies that adolescent mothers with HIV are more likely to be discriminated against, either regarding the teenage pregnancy itself or from the HIV infection. To tackle the stigmatization and discrimination issues among these groups of people living with HIV, this study was conducted to gather more information about their experience during pregnancy while they are living with HIV during their adolescence.

The primary objective of this study was to explore how HIV-positive pregnant women managed to live through stigmatization using coping mechanisms. The secondary objective was to determine factors that contribute to stigma during their motherhood as well as their response to day-to-day discrimination.

\section{Methodology}

A study using the qualitative method was deployed in this study. Face-to-face in-depth interviews were conducted using interview guidelines to collect data from pregnant adolescents living with HIV. To prevent the volunteers from trauma during pregnancy, the researcher tried to avoid sensitive questions. The interview was carried out with 16 volunteers.

The research instrument consisted of 14 questions related to pregnancy, antenatal care, HIV infection history, disclosure of HIV status, the stigmatization experience and problem management. The volunteers were free to request that any questions be skipped if they felt uncomfortable, or to ask that the interview be terminated at any time at their convenience.

\section{Informants}

The respondent group included all women expecting a child who visited the reproductive health clinic at Chulalongkorn Hospital and the HIV-Netherlands Australia Thailand 
Research Collaboration Center during the second year of data collection in 2019. A purposive sampling approach was used to identify and recruit eligible participants.

\section{Data analysis}

Data analysis was used to gather preliminary findings and analyze the data using research questions and the theory discussed to collect and summarize ideas from experiences of HIV-related stigma interpretation, contemplation and reflection during data collecting. We also made plans for greater data collection. No new or unusual data were gathered until sufficient saturation of data had been achieved from the field. The data-gathering procedure aimed to detect and address the problem of pregnant women living with HIV. Themes of stigma experiences and coping methods were captured using content analysis.

\section{Ethical consideration}

Ethical consideration was approved under the committee of the Institutional Review Board, Faculty of Medicine, Chulalongkorn University (IRB. \# 655/61; date of approval: 17 Jan. 2019).

\section{Results}

Of the 50 pregnant women living with HIV who visited the clinic, 16 were approached and recruited to participate in this study to provide in-depth information to the point of saturation by interview method.

\section{Demographic data}

A total of 16 participants were divided into two groups according to their age when they first discovered their pregnancy. The first group consisted of five participants who became pregnant during adolescence. Regarding their educational level, two $(40 \%)$ of the total five participants stated that their highest education was primary school level, while two $(40 \%)$ others completed secondary school and only one (20\%) participant was a university student at the time her pregnancy was discovered. In terms of marital status, $60 \%$ of the participants were living with their partners when they got pregnant, while $40 \%$ of them were separated. We examined how they discovered their HIV-positive test result, and most of them, accounting for $60 \%$, reported having learned about their infection status during a visit for antenatal care services, while only one person stated that she discovered her status during a regular HIV check-up. The possible cause of HIV infection was also investigated in this study among teenagers with pregnancy who live with HIV; the main route of transmission was sexual transmission $(60 \%)$ and unknown cause ( $40 \%)$. It is noteworthy that all participants in this study had disclosed their HIV status to their families.

Experiences of stigmatization. It can be quite difficult and overwhelming for a woman to learn of her HIV infection, especially during pregnancy [9]. One of the most prevalent concerns among these women is the risk of transmitting the virus to their babies, which subsequently leads to the dilemma of receiving HIV treatment but with the possibility of disclosing their infection status before they wished to do so. However, the biggest challenge of all was determining how to reveal their infection status to family members without shocking them. For that reason, many mothers did not want to reveal their infection status to their husbands for fear of losing support from their family and experiencing other negative consequences such as domestic violence.

The results of this study concerning the feelings that these women experienced after learning of their HIV infection were analyzed as follows:

Being mothers living with HIV: perception of the first known diagnosis of HIV infection. The interview process revealed different perceptions and points of view among our 
JHR

36,6

1144

participants. Based on all perspectives, most of the information the respondents had about HIV infection came from health care providers such as doctors who provided counseling about their HIV status. Moreover, most teenage mothers lived with their families who provided financial support. In addition, for teenage clients to proceed with HIV treatment, their parents were required to be notified of the treatment plan. Therefore, teenage mothers who had HIV were required to disclose their infection status to their parents. This could be an advantage of providing care to pregnant teenagers who are HIV positive, but at the same time, the young mothers' primary source of information about HIV treatment was doctors who made it clear that HIV patients are required to take life-long medication to prevent mother-to-child transmission. Again, this might cause different outcomes between adolescent mothers and adult mothers in terms of perception of HIV infection.

HIV-status disclosure among young mothers living with HIV: disclosure to partners and family members. It is common for adolescents to reveal their HIV-positive status to parents first. This is because teenagers can feel overwhelmed, and they probably have no one else whom they can trust and who can give them support for treatment and help raise their babies. The following information reflects what the respondents of this study revealed.

At first, I do not know what to do, so I told my mother and my boyfriend. I was so lucky that my mom supports me through my difficult time. I would say my mom is everything to me. She is very supportive. (Participant 16, 20 years old)

At that time, doctors disclosed to me and my family at the same time. They were upset too, but they were good to me as always, they did not act differently at all. My mother helps me raise my child. (Participant 12, 17 years old)

\section{Stigmatization among pregnant teenagers living with HIV: Feelings of anxiety}

Pregnancy among teenagers is normally unwanted and unexpected. Most girls are in school, and they often do not know how to deal adequately with this unexpected situation. Therefore, it is likely that they will seek an abortion. However, with social support, they cope with their pregnancy.

When I knew that I got pregnant, I was afraid that I would lose my youth, I was anxious about all things that could happen. I was afraid that this will upset my parents. However, I decided to tell my parents, in return I received their support in every aspect. I started to see things clearly and I moved on with my life easier. (Participant 13, 16 years old)

HIV-status disclosure among adult mothers living with HIV. Non-disclosure to family: Due to possible negative consequences, some study participants decided not to tell anyone about their HIV status.

Until now, I did not tell anyone in my family about my HIV status. I am afraid that they will hate me and my child, so I keep it a secret. If I would have to tell anyone, I would tell my husband. I still think that other people see this disease as disgusting. (Participant 10, 39 years old)

I did not tell anyone because I broke up with my husband before knowing that I was infected with the disease. Afterwards, I take care of myself and my child alone. (Participant 5, 25 years old)

Disclosure to partner: Some mothers chose to tell only their husband, because they did not want to risk disclosing this sensitive information to other people and because they were certain that their husband was the source of their HIV infection. If they had a healthy relationship with their husband, it was not necessary to tell others.

I was very worried, but after I told my husband, I was supported a lot by my husband. Moreover, doctors also provide support in terms of ensuring that if I take medicine our child would not infect with the disease. My husband is very supportive. (Participant 8, 27 years old) 
However, not every situation was resolved so smoothly. In some families, when the young mothers told their husbands the truth about why they could not maintain a healthy relationship, it resulted in negative consequences, especially in serodiscordant couples.

I told my husband, but he doubted me, and he was not infected with HIV so he left me since then. (Participant 9, 32 years old)

Experience of being socially discriminated against from HIV infection during pregnancy. In addition to facing HIV infection, mothers experience worries regarding their pregnancy. This could create more concern among them about whether they should tell anyone or how they can live without social support. However, this depends on individual experiences. If they faced negative social criticism, the individuals might feel discriminated from society, while some mothers who received positive support from their social networks experienced help through their difficult times.

I have a good experience during my pregnancy to term; the doctors provided useful information. I can feel they treated me the same as other people. I think this is very helpful and very supportive to me. (Participant 7, 27 years old)

Coping mechanisms. It is understandable that, after learning about their HIV status, people coped in different ways depending on their social context and perception. This study shows different methods employed by the subjects to cope with their health issues.

\section{Coping mechanism among pregnant teenagers living with HIV: Receiving great social support from parents}

Parents are key figures who help teenage mothers get through hard times. If these vulnerable subjects do not have support and understanding from their family, they face a lower quality of life.

At first, I was worried so much, but after I told my parents and my boyfriend, they treated me as normal, nothing changed, and they accepted the reality. (Participant 13, 16 years old)

My family helps me a lot, they cheer me up, they take really good care of me, and they understand me. If I did not have a very supportive family, I would not be able to get through this. (Participant 12, 17 years old)

Regarding teenage mothers who were still in school, their life achievement still included completing school to live their lives fully after giving birth. Therefore, some teenage mothers still see it as important to them to be back in school after giving birth.

I think I am going to be back to school and complete at least high school in order to find a better job to raise my child. (Participant 12, 17 years old)

Coping mechanism among adult mothers living with HIV: As a result of this study, we found that parents are key elements for adult mothers living with HIV to achieve positive outcomes. Even though adult mothers are more likely to be self-reliant and are also more responsible than teenage mothers, they still need support from family, especially their partners.

I think the most important thing helping me to get through this difficult time is encouragement from my family, especially my husband. He is very supportive, and he takes very good care of me. (Participant 8, 27 years old)

Some adult mothers find their own ways to reduce their stress. Letting things go was one of many ways that they endured their suffering.

I just do not overthink, I let it be. I just followed the doctor's suggestions and took good care of myself when I was pregnant. That was quite a help to me until I gave birth. (Participant 6, 25 years old) 
JHR

36,6

1146

\section{Discussion}

The study results revealed that stigmatization among pregnant women people living with HIV is not only influenced by age but also other external factors. Other factors included source of income, duration of HIV infection and most importantly, support from family [10]. Therefore, family support is vital to help HIV-positive pregnant women overcome stigmatization and depression during motherhood. The result from this study was similar to those of Paudel and Baral [6] and Brittain et al. [11], which suggested that people who succeed in dealing with the problem of HIV status disclosure were most often those who had strong family and social support. The previous study suggested that most teenagers who disclosed their HIV status to family were more likely to have access to medical treatment. In general, family support played a vital role in helping teenagers to take care of themselves during motherhood. One of the reasons teenagers need support from their parents is that they are not mature enough to see problems thoroughly. Moreover, their naivety could possibly lead to less internal stigmatization. In comparison to teenage mothers, it was found that adult mothers were less likely to reveal their HIV status during pregnancy. The most common reason for this is probably the fact that they are financially and emotionally independent of their parents. These women tended to reveal their HIV status to their partner/husband [11, 12]. This leads to the assumption that pregnant women living with HIV are afraid to reveal their HIV status. Our results contrast with the study by Li et al. [4], which studied HIV stigmatization in rural areas in China. Their result showed that young mothers are more likely to experience stigmatization than mature mothers. However, the similarity between that study and ours is that less-stigmatized mothers received more support from family and society. Another similarity is that teenage mothers received support mostly from parents [4]. For this reason, stigmatization affects quality of life, physical health and mental health [13].

Personal stigma affects pregnant women living with HIV of all ages. Teenage mothers experienced the most worry regarding the need to take life-long medication as a result of their infection [14-16]. Adult mothers faced the major concern about how much longer they would live. However, one concern that the two groups of mothers have in common was the impact on their children [17]. This finding has shown a similar result to that of Ion et al. [18]. That study explored "HIV-related stigma in pregnancy and early postpartum of mothers living with HIV in Ontario, Canada" with the result suggesting that stigmatization can cause HIV-infected mothers to experience stress as a result of HIV infection and pregnancy.

Negative self-image was found amongst some of the participants, especially among adult mothers. These people hold the perception that those infected with the disease are not hired for work and cannot live in society and that people with HIV have wounds and a bad physical appearance. This perception depends on personality type and how each person understands the world.

Regarding social stigma, both groups of participants reported that social stigma was the cause of their low self-esteem and low self-value. They were afraid of being treated differently from other people in society $[6,19]$. Regarding social attitudes related to stigmatization, HIVpositive teenage mothers were less stigmatized than their older counterparts. Once women living with HIV found out about their pregnancy status, many developed stress, depression, and other negative emotional consequences, which led them to cover up their HIV status from their husbands. One obvious situation was found among teenagers living with HIV who become adults. They were afraid to attend social gatherings because of the external stigmatization and discrimination from those in their workplace. Moreover, they feared that their HIV-positive status could alienate them from other colleagues and eventually lead to job loss. The fear among people living with HIV is based on the feeling that they are often compared to those with horrifying physical appearances as a result of AIDS, which corresponds to the study of Simbayi et al. [7]. Their study was conducted among HIV-infected patients who visited a clinic in Cape Town [7]. This study indicated that $40 \%$ of them were discriminated against, which led to difficulties functioning within their society. 
Regarding coping mechanisms [20], people who had social support, especially family and significant others, were found to be able to cope and endure their difficulties better than those who lacked support. Support from health care workers is also essential to those who experience stigma [21]. Health care personnel can be a source of empowerment to those who have trouble with this situation. This study yields similar outcomes to several other studies conducted in Thailand and other countries in that stigmatization and discrimination are still present in our society. HIV-infected pregnant women were found to experience higher levels of personal stigma. However, on a more positive note, this study revealed that family and social support [2] were the most important factors in helping these women endure their difficulties. This qualitative study sought to establish a policy and further campaign to minimize stigma among pregnant teenagers living with HIV, and such policies should focus on social support. Family members should be encouraged to play a vital role in supporting these vulnerable people.

\section{Conclusion}

This study found that personal stigma is still present among pregnant women of all ages who are living with HIV. One of the remaining challenges for those who are stigmatized is the disclosure of their HIV status. These women's HIV status was kept a secret. Surprisingly, all adolescent mothers who took part in this study told their families about their HIV status, including their husbands, parents and husband's relatives. People who have social support, particularly parents and significant others, have been proven to be able to cope and overcome hardships better than those who do not.

Conflict of Interest: None

\section{References}

1. Pannetier J, Lelievre E, Le Coeur S. HIV-related stigma experiences: understanding gender disparities in Thailand. AIDS Care. 2016; 28(2): 170-8. doi: 10.1080/09540121.2015.1096888.

2. Brittain K, Mellins CA, Phillips T, Zerbe A, Abrams EJ, Myer L, et al. Social support, stigma and antenatal depression among HIV-infected pregnant women in South Africa. AIDS Behav. 2017; 21(1): 274-82. doi: 10.1007/s10461-016-1389-7.

3. Churcher S. Stigma related to HIV and AIDS as a barrier to accessing health care in Thailand: a review of recent literature. WHO South East Asia J Public Health. 2013; 2(1): 12-22. doi: 10.4103/2224-3151.115829.

4. Li Z, Morano JP, Khoshnood K, Hsieh E, Sheng Y. HIV-related stigma among people living with HIV/ AIDS in rural Central China. BMC Health Serv Res. 2018; 18(1): 453. doi: 10.1186/s12913-018-3245-0.

5. Mchunu G, Peltzer K, Tutshana B, Seutlwadi L. Adolescent pregnancy and associated factors in South African youth. Afr Health Sci. 2012; 12(4): 426-34. doi: 10.4314/ahs.v12i4.5.

6. Paudel V, Baral KP. Women living with HIV/AIDS (WLHA), battling stigma, discrimination and denial and the role of support groups as a coping strategy: a review of literature. Reprod Health. 2015; 12: 53. doi: 10.1186/s12978-015-0032-9.

7. Simbayi LC, Kalichman S, Strebel A, Cloete A, Henda N, Mqeketo A. Internalized stigma, discrimination, and depression among men and women living with HIV/AIDS in Cape Town, South Africa. Soc Sci Med. 2007; 64(9): 1823-31. doi: 10.1016/j.socscimed.2007.01.006.

8. Salters K, Loutfy M, de Pokomandy A, Money D, Pick N, Wang L, et al. Pregnancy incidence and intention after HIV diagnosis among women living with HIV in Canada. PLoS One. 2017; 12(7): e0180524. doi: 10.1371/journal.pone.0180524.

9. Goffman E. Stigma. Englewood Cliffs, New Jerssey, NJ: Prentice-Hall; 1964.

10. Arshi M, Yavari M, Fekr Azad H, Safi MH, Moghanibashi-Mansourieh A, Moshayyedi M. Investigation of relationship between family social support and the level of stigma perceived by PLWHA in Iran. Soc Work Public Health. 2020; 35(3): 90-9. doi: 10.1080/19371918.2020.1742840. 
JHR

36,6

1148
11. Brittain K, Mellins CA, Remien RH, Phillips TK, Zerbe A, Abrams EJ, et al. Impact of HIV-status disclosure on HIV viral load in pregnant and postpartum women on antiretroviral therapy. J Acquir Immune Defic Syndr. 2019; 81(4): 379-86. doi: 10.1097/QAI.0000000000002036.

12. Rogers AJ, Achiro L, Bukusi EA, Hatcher AM, Kwena Z, Musoke PL, et al. Couple interdependence impacts HIV-related health behaviours among pregnant couples in southwestern Kenya: a qualitative analysis. J Int AIDS Soc. 2016; 19(1): 21224. doi: 10.7448/IAS.19.1.21224.

13. Rueda S, Mitra S, Chen S, Gogolishvili D, Globerman J, Chambers L, et al. Examining the associations between HIV-related stigma and health outcomes in people living with HIV/AIDS: a series of meta-analyses. BMJ Open. 2016; 6(7): e011453. doi: 10.1136/bmjopen-2016-011453.

14. Makoae LN, Portillo CJ, Uys LR, Dlamini PS, Greeff M, Chirwa M, et al. The impact of taking or not taking ARVs on HIV stigma as reported by persons living with HIV infection in five African countries. AIDS Care. 2009; 21(11): 1357-62. doi: 10.1080/09540120902862576.

15. Katz IT, Ryu AE, Onuegbu AG, Psaros C, Weiser SD, Bangsberg DR, et al. Impact of HIV-related stigma on treatment adherence: systematic review and meta-synthesis. J Int AIDS Soc. 2013; 16(3 Suppl 2): 18640. doi: 10.7448/IAS.16.3.18640.

16. Peltzer K, Ramlagan S. Perceived stigma among patients receiving antiretroviral therapy: a prospective study in KwaZulu-Natal, South Africa. AIDS Care. 2011; 23(1): 60-8. doi: 10.1080/ 09540121.2010.498864.

17. Volmink J, Marais B. HIV: mother-to-child transmission. BMJ Clin Evid. 2008; 2008: 909.

18. Ion A, Wagner AC, Greene S, Loutfy MR. HIV-related stigma in pregnancy and early postpartum of mothers living with HIV in Ontario, Canada. AIDS Care. 2017; 29(2): 137-44. doi: 10.1080/ 09540121.2016.1211608.

19. Liping M, Peng X, Haijiang L, Lahong J, Fan L. Quality of life of people living with HIV/AIDS: a cross-sectional study in Zhejiang province, China. PLoS One. 2015; 10(8): e0135705. doi: 10.1371/ journal.pone.0135705.

20. Folkman S. Stress: appraisal and coping. In: Gellman MD, Turner JR, editors. Encyclopedia of ehavioral medicine. New York, NY: Springer; 2013. p. 1913-5.

21. Alexandra Marshall S, Brewington KM, Kathryn Allison M, Haynes TF, Zaller ND. Measuring HIV-related stigma among healthcare providers: a systematic review. AIDS Care. 2017; 29(11): 1337-45. doi: 10.1080/09540121.2017.1338654.

\section{Corresponding author}

Narin Hiransuthikul can be contacted at: narin.h@chula.ac.th

For instructions on how to order reprints of this article, please visit our website:

www.emeraldgrouppublishing.com/licensing/reprints.htm

Or contact us for further details: permissions@emeraldinsight.com 